\title{
ICT tools for enabling smart grid players' flexibility through VPP and DR services
}

\author{
George Messinis \\ Aris Dimeas \\ Nikos Hatziargyriou \\ Dept. of Electrical and Computer Engineering \\ National Technical University of Athens \\ Athens, Greece
}

\author{
Isidoros Kokos \\ Ilias Lamprinos \\ Telco Software Dpt. \\ Intracom S.A. Telecom Solutions \\ Athens, Greece
}

\begin{abstract}
The European plan on climate change consists of a range of measures to fight against climate change, one of which is to reach $20 \%$ of renewable energy in the total energy consumption in the $\mathrm{EU}$ by $\mathbf{2 0 2 0}$. The increasing penetration of RES and DG introduces new business cases which require innovative ICT tools and the support of appropriate communication infrastructure. This paper focuses on the Virtual Power Plant and Demand Response cases, proposing a general architecture as well as analyzing core functionalities, information and communication requirements, along with relative standards and technologies. The architectural analysis is based on the SGAM framework, a standardized architecture for smart grid solutions and services that allows new distribution grid players to participate in the energy markets and contribute to an optimal grid operation. The document presents the realized use cases' modeling and gives an overview of the pilots in which the proposed models will be materialized and validated.
\end{abstract}

Index Terms - Demand Response, SGAM, Smart Grid Services, Virtual Power Plant

\section{NEW SERVICES For THE SMART DistribUtiON GRID}

One of the most important changes included in the new smart grid paradigm is the large scale integration of heterogeneous and geographically dispersed distribution grid resources like DERs, EVs, energy storage systems and smart loads. Such resources could be used both for supporting the operation of the distribution grid and in market participation applications. Currently, the use of distribution grid resources in these application domains is rather limited due to the existing energy market and regulatory frameworks and the lack of appropriate ICT architectures.

However, the emerging energy market deregulation together with the advent of smart grid oriented communication and information technologies enable new business players to provide the aforementioned services (i.e. market participation and optimal distribution grid operation) to already existing actors (i.e. producers, consumers and the DSOs). These new smart grid players, frequently referred to as Aggregators, have the ability to integrate various distribution grid resources. A new business model is thus being formed in the distribution domain which apart from supporting most of the previously passive distribution grid components and actors introduces new active market participants also able to take part in the grid operation optimization. Two such entities are the Virtual Power Plant and Demand Response Aggregators. Their role and associated ICT infrastructure are of main interest in this paper.

A lot of work has been conducted on the Virtual Power Plant concept [1]. This work includes a lot of academic research, proposing various algorithms for VPP operation considering both technical and market operation [2]. In addition, a number of EU projects implemented exemplary VPPs and demonstrated their pilot operation [3]. However, in every case the VPP is defined in a different and frequently obscure way making it difficult to understand basic concepts such as the participating actors and components, required functionalities, communication and information requirements. A standardized description of a specific VPP architecture may provide an answer to the aforementioned problem and foster market adoption of the VPP concept and its relative solutions.

On the other hand, the concept of Demand Response [4] is now considered quite elaborated since a lot of work has been conducted not only as academic research but also towards commercialization. It is widely explored in EU projects and a lot of initiatives exist to transfer relevant technologies in real environments [5]. However, apart from pilots, there is still no example of large scale implementations of DR by a DSO, which again proves that there are still a lot of barriers to overcome. The absence of a concrete and sound use case (both for VPP and DR) analysis that will lead to easily adopted and reusable ICT architectures is one of these barriers.

VPP and DR are considered to be of utmost importance for market oriented applications as well as for distribution grid management applications. In the previous paragraphs a number of references were given for the two use cases, which indicate that there is a lot of synergy between them. In fact, the case of a VPP that includes a large number of loads [6], [7] can be viewed as a VPP interacting with an internal DR service. Thus, any proposed smart grid related ICT architecture should incorporate this kind of interaction. 


\section{THE SMARTEREMC2 PROJECT}

\section{A. Project overview and goals}

The SmarterEMC2 [8] project aims at linking several industrial and academic groups in different areas of ICT systems and power systems with a common goal of achieving a breakthrough in the ICT integration with power systems for enhancing various smart grids services. The main objective of the project is to propose business models and to develop the necessary ICT tools to support Customer Side Participation (CSP), increase RES penetration, and foster the participation in the electricity market. The SmarterEMC2 project has the following ambitious objectives:

- Definition of new business cases and roles supporting the integration of consumers in the electricity market.

- Definition of new business cases and roles supporting the integration of DG/RES.

- Definition of new services for smart grids enabling all actors involved in the management and operation of the Distribution Grid

- Demonstration of innovative ICT tools for consumption and production forecasting, for microgrid management, and for data analytics and big data management.

- Evaluation of the current communication infrastructure through hardware and software simulation and assessment of the feasibility of using this infrastructure in the emerging smart grid services.

- Piloting and validation of innovative ICT solutions in laboratory and real life environments

- Exploration of emerging business models in the smart grid domain.

\section{B. The SmarterEMC2 use cases}

Fig. 1 presents the SmarterEMC2 high level conceptual architecture, with all relevant components to be developed in the project. Each component is mapped to specific use cases, as presented in short below:

- Virtual Power Plants: In this case VPP participation in the wholesale and intraday markets is considered as well as the provision of ancillary services like voltage control.

- Demand Response: The DR use case includes demand response dispatch for grid operation and market participation, as well as DR resources management.

- Local Constraints Management: In this set of use cases the DSO identifies distribution grid problems, defines preventive control actions and adjusts flexibility offers to ensure optimal grid operation.

- Energy Hub: The Energy Hub includes a set of functionalities related to smart microgrid operation, including optimal scheduling, monitoring and control of distributed flexibility assets.
In order to support the aforementioned use cases, a data analytics platform is designed with the main goal to provide forecast, visualization and data handling services to other components of the above mentioned use cases.

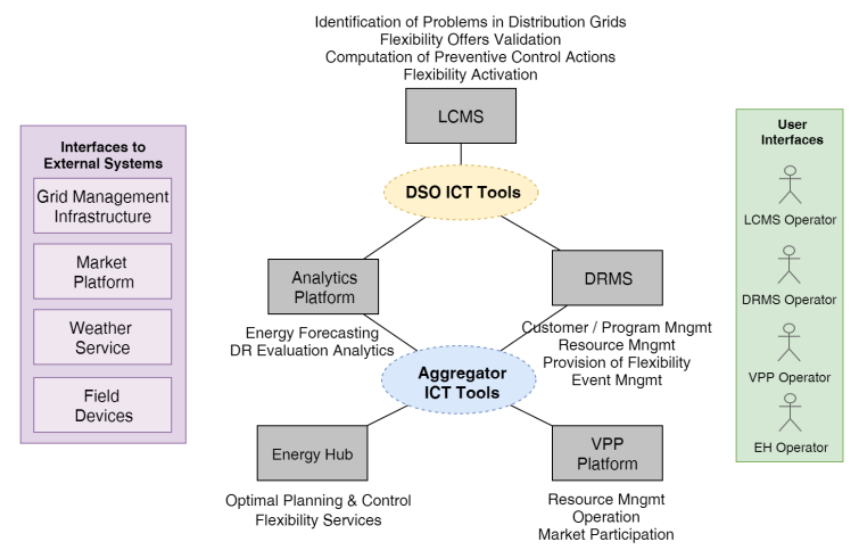

Figure 1. The SmarterEMC2 High Level Conceptual Architecture

\section{Modelling use cases with SGAM}

The use cases mentioned above require information from a large number of application domains (DER, electricity market, distribution network, demand response resources etc). The Smart Grid Architecture Model (SGAM) [9] is used to analyze the use cases and extract system requirements and specifications. Apart from providing a standardized modeling procedure, SGAM is also a great tool for extracting interoperability requirements on various levels, namely communication, information, function and business. This procedure is extremely important when facing the task of implementing a real life system, like a VPP Platform or a DR Management System (DRMS) where a lot of different components need to communicate, exchange messages and coordinate their functionalities in order to achieve a greater goal. SGAM has not been yet widely adopted for analyzing smart grid use cases. Nonetheless a good amount of published work demonstrating the use of the model exists in literature [10]-[13], so no further analysis will be presented here apart from the high level use case modeling procedure followed in the project (Fig. 2). In this iterative procedure the modeler first defines a business case and relevant high level use cases (HLUCs) to achieve business goals. Each HLUC is broken down to primary use cases (PUCs) and PUC actors (as well as their interactions) are defined. This procedure is repeated until a satisfactory level of detail is achieved. Next the SGAM layers are developed which typically leads to revising the initial modeling assumptions (PUCs, actors etc).

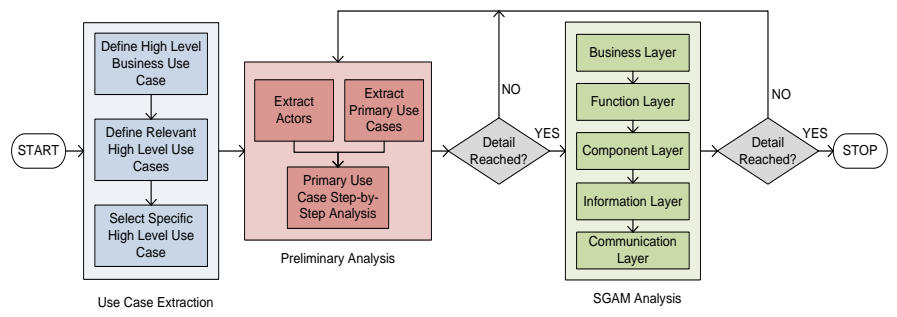

Figure 2. The SmarterEMC2 methodology for SGAM use case modeling 


\section{DEMAND RESPONSE AND VIRTUAL POWER PLANTS IN THE DISRTIBUTION GRID}

In this chapter the SmarterEMC2 approach to the Virtual Power Plant and Demand Response use cases is presented using the SGAM framework. The focus is on the function, information and communication layer, while the reader can also see the component layer on the background of the other three layers. The business layer is not presented here since the intention of the authors is to present a generic technical solution compliant with the prevalent business cases.

\section{A. Functional Requirements}

Fig. 3-4 present the SGAM Function Layers of VPP and DR use cases, whilst also present use cases related to DSO's operations for grid management. The depth of functional decomposition was carefully chosen in order to make sure that the resulting model actually supports the application design procedure without making it more complex. Thus, the basic functionalities of an Aggregator (either VPP or DR) participating in the energy markets or providing ancillary services to the DSO are described using VPP and DR concepts as modeled by the VPP Operator and DRMS components, respectively. The aggregator uses forecast information and knowledge of its units' flexibility in order to calculate its flexibility and place market bids. After market closure the Aggregator follows the market schedule by planning and dispatching control schedules to its units, in the case of VPP, or events in the case of DR. In the case of VPP a real time monitoring operation adjusts the units' production/ consumption in real time in order to compensate for forecast errors and unexpected events whilst in the case of DR,

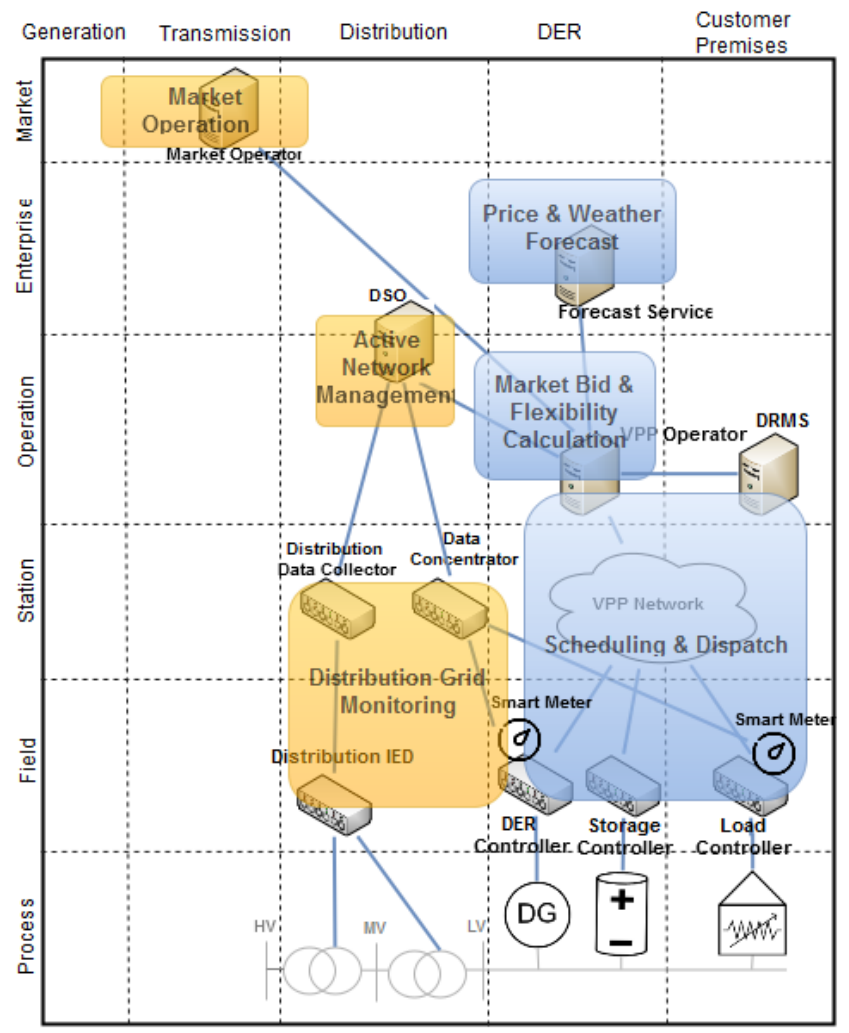

Figure 3. Virtual Power Plant Function Layer specific programs such as Direct Load Control can be selected for compensating poor participation results.

Market Operation: This functionality allows the Aggregator to participate in the wholesale and intraday markets by placing bids. The functionality of placing a market bid in a market platform is not a trivial task, although it is significant to identify message exchanges and interactions between actors that are not currently able to participate.

Price \& Weather Forecast [14]-[16]: It is widely accepted that forecasting is one of the most important functionalities that must be available on the distribution level in order to enable a more reliable and efficient operation of distribution grid actors such as the VPP or DR Aggregator. Forecast algorithms both for day-ahead PV and wind energy production are available and facilitate the VPP operation. In addition, load forecast algorithms are of utmost importance for the operation of the DR Aggregator, mainly by providing estimations of future aggregated consumption. These estimations can be used to calculate market bids and to optimally schedule units (DERs, storage, loads) and dispatch them.

Market Bid \& Flexibility Calculation [17]-[19]: A core functionality of the Aggregator (VPP, DR) is the calculation of optimal market bids that is based on the flexibility estimation of the units. These are two different, though connected tasks that need to be carefully designed. Calculating market bids or, with other words, designing the best market participation strategy can be a devious task for an Aggregator. The possibilities are numerous and mainly depend on the market structure. Either way, in order to

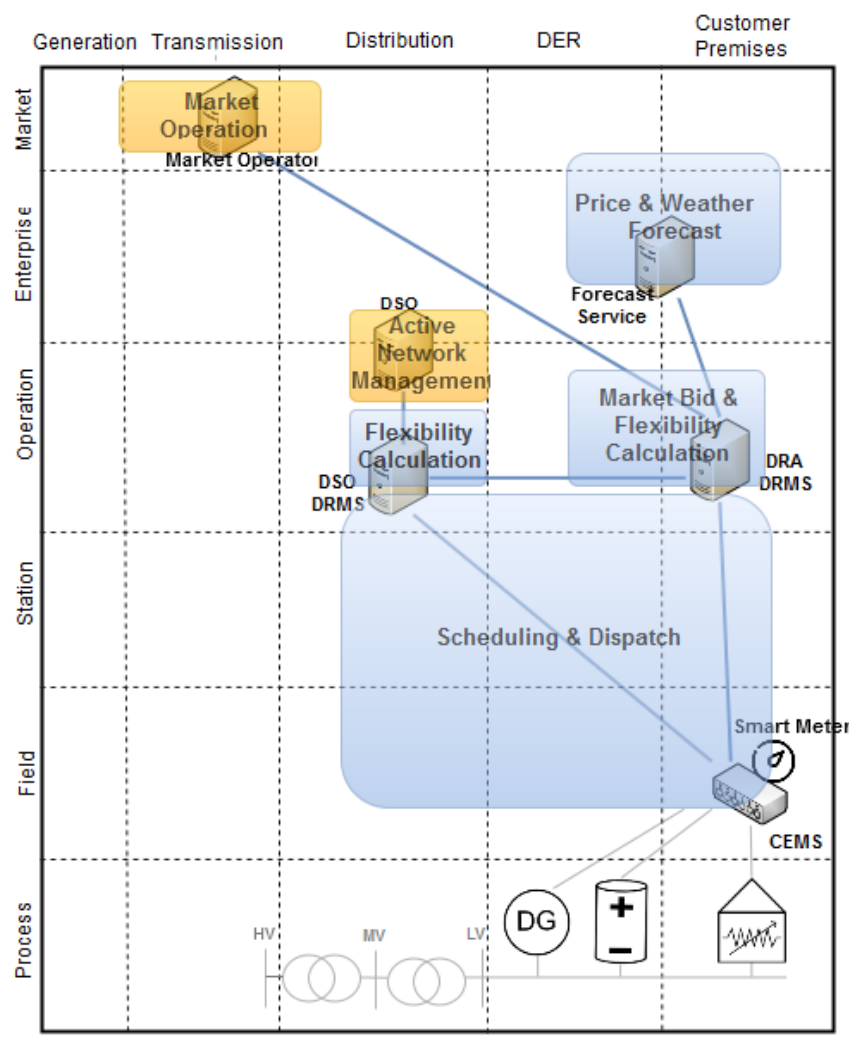

Figure 4. Demand Response Function Layer 
calculate optimal bids one has to have a good estimation of unit's flexibility, that is for example technical restrictions on the unit's ability to change its power consumption or production. This might be easy to define in the case of a PV or diesel generator but might be a much more difficult task in the case of residential consumers. Fig. 4 presents the case where the DSO operates a DRMS for managing DR resources, therefore has a need for estimating the available flexibility.

Scheduling \& Dispatch [20]-[24]: This is another core functionality that the SmarterEMC2 project focuses on by developing relevant algorithms. Optimal scheduling (e.g. day ahead) of the VPP as well as real time dispatch in order to compensate for scheduling errors can be implemented with a number of algorithms. Our approach is the decentralized dispatch, where a multi-agent system is installed on the local controllers and solves the dispatch/scheduling problem by message exchange between agents.

DG monitoring \& Active Network Management: Fig. 3-4 present (in yellow) DSO functionalities that need to take place in order to trigger a VPP (or DR) Aggregator for providing ancillary services. In this case the DSO needs to be able to locate and detect problems in the distribution network ("Distribution grid monitoring") and take the optimal measures to solve them("Active Network Management"), including the dispatch of relative VPP units or DR resources.

\section{B. Information \& Communication Requirements}

Fig. 5-6 present the information and communication layer of VPP and DR use cases correspondingly. This analysis is of high significance for identifying information and communication requirements. Communication solutions and protocols, as well as information standards are listed here in order to reveal interoperability issues between components

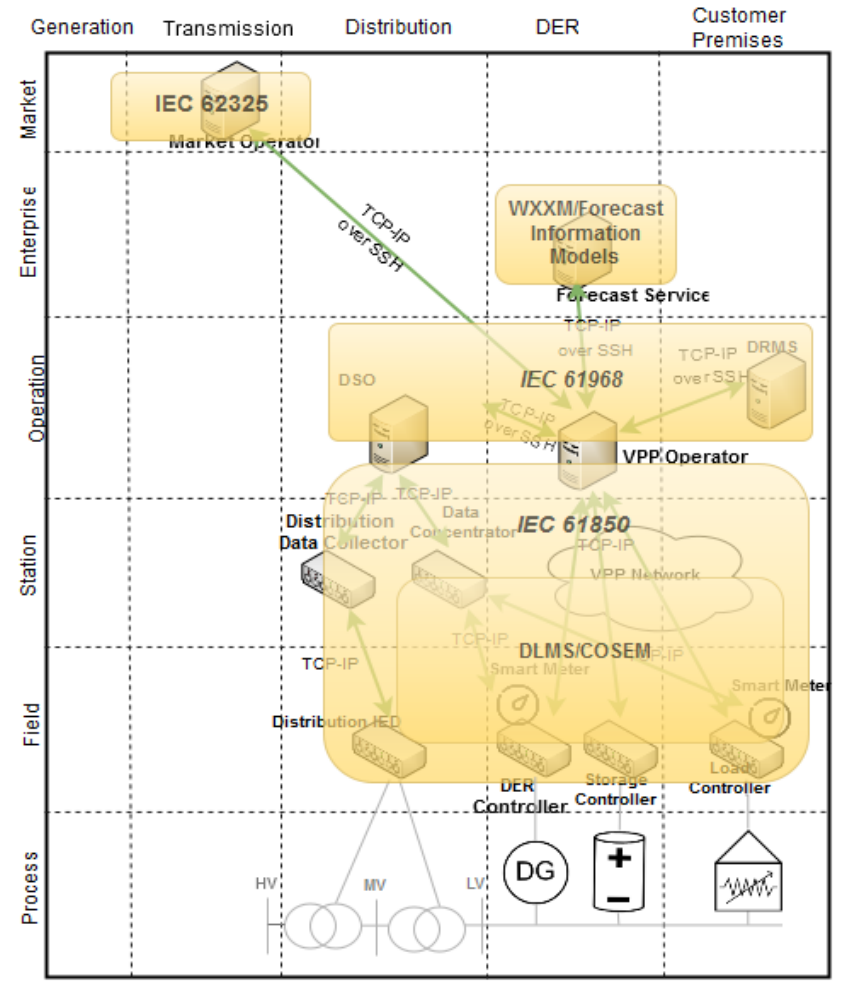

Figure 5. VPP Information \& Communication Layers and actors. For the case of VPP, the IEC 61850 standard was selected for information exchanges in the Process, Field and Station zones, since it is an industrial standard that covers the distribution and DER domains whilst lot of work for its expansion is currently taking place [11]. The standard includes a large set of data models describing a vast amount of information managed by devices operated by the VPP. On the contrary, the DRMS utilizes the IEC 62746 standard and specifically Part 10-1: Open Automated Demand Response. Originally designated as OpenADR $2.0 \mathrm{~b}$ profile specification [25], the standard provides flexible data models as well as transport mechanisms to facilitate common information exchange between smart grid actors and has been deployed in several DR projects. IEC 61968 is used for interaction with DSO's infrastructure, in cases where the DSO needs to access the Aggregator's infrastructure and/or services, or in case the Aggregator requires access to DSO's data (e.g. energy measurements). In addition, DLMS/COSEM, the most popular smart metering standard, is also required by the VPP in case direct access to smart meters is assumed. Another CIM standard, IEC 62325 which describes message exchanges between market actors, is adopted for market participation.

Forecasting assumes an important role in such applications, hence forecasting related standards are considered, especially in the case where external actors provide forecasting services to Aggregators. Weather forecast standards already exist (for example WXXM) [26]. In contrast, non proprietary standards for energy forecast are more difficult to find.

Finally, communication requirements may also be defined by describing the communication links between various components. This kind of analysis though is bound to the

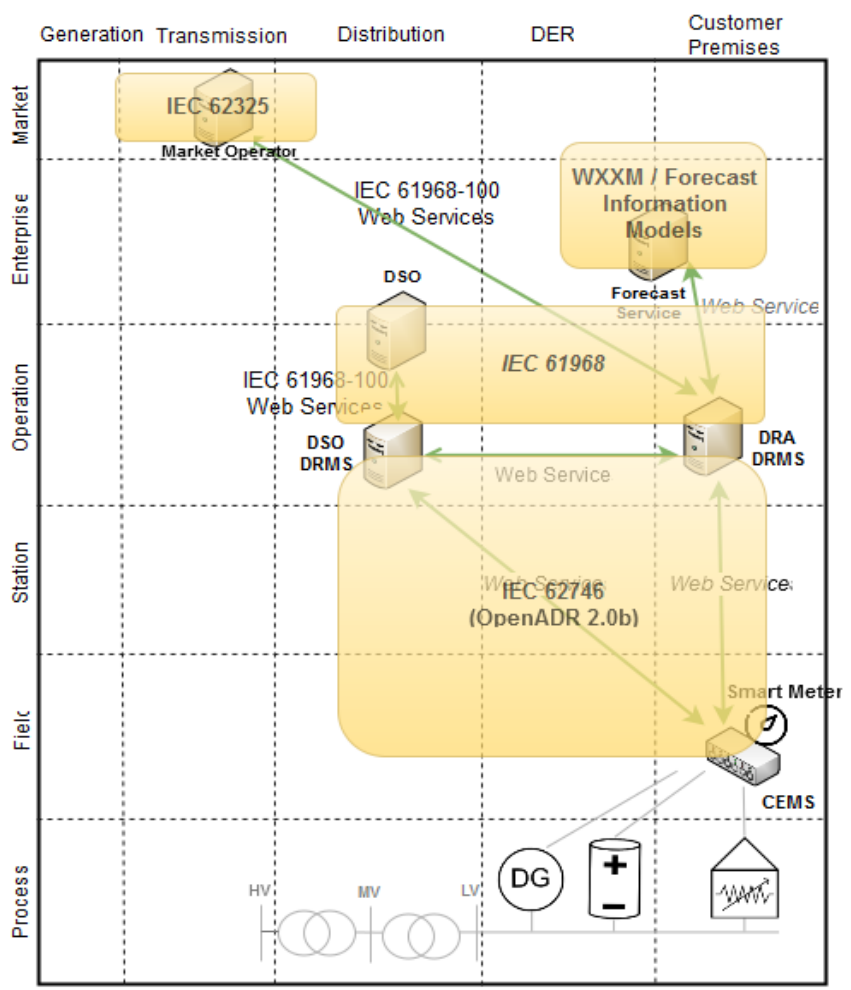

Figure 6. Demand Response Information \& Communication Layers 
final implementation' restrictions (especially choosing communication technologies), having to do with existing infrastructure, distance, site etc. In the case of VPP a general choice of TCP/IP based communication protocols can be considered legitimate, while securing external communication links (for example the VPP-DSO, VPPForecast Service or VPP-Market Operator) by choosing TCP/IP over SSH is common sense. In our approach, the DR use case incorporates the use of web services as a high level communications protocol and investigates the IEC61968-100 standard for the communication of the DRMS with DSO, Forecast Service and the Market Operator.

\section{CONCLUSIONS AND NEXT STEPS}

This paper describes the SmarterEMC2 approach in defining ICT oriented smart grid use cases like the Virtual Power Plant and Demand Response. The activities carried out thus far have led to the definition of the functional and technical requirements and the overall conceptual architecture of an ICT ecosystem that supports novel smart grid services. The defined functionalities are currently being studied in order to design the most suitable algorithms for DR and VPP operation. Information requirements (extracted both from the information layer and function layer by a step-by-step analysis of the functionalities) are used in order to define the pilot's data repository structure as well as any required interfaces for sending/receiving information objects. In conclusion, the presented SGAM use case analysis has been extremely helpful by providing a more systemic view of the application, exposing a number of interoperability and operation issues that would otherwise endanger the course of the project. The ICT tools presented in this paper will be eventually implemented, integrated and demonstrated in the "Meltemi" pilot site and the NTUA microgrid laboratory [27], Athens, Greece. The Demand Response platform will be demonstrated also in a pilot site in Turkey.

\section{ACKNOWLEDGEMENTS}

This work was supported in part by the European Community's Horizon 2020 Framework Program under grant agreement No 646470, SmarterEMC2 Project. The authors would like to thank their colleagues in SmarterEMC2 project for their collaboration.

\section{REFERENCES}

[1] G. Plancke, K. De Vos, R. Belmans, and A. Delnooz, "Virtual power plants: Definition, applications and barriers to the implementation in the distribution system," Int. Conf. Eur. Energy Mark. EEM, vol. 2015-Augus, 2015.

[2] P. Moutis and N. D. Hatziargyriou, "Decision trees-aided active power reduction of a virtual power plant for power system overfrequency mitigation," IEEE Trans. Ind. Informatics, vol. 11, no. 1, pp. 251-261, 2015.

[3] C. Kieny, B. Berseneff, N. Hadjsaid, Y. Besanger, and J. Maire, "On the concept and the interest of virtual power plant: Some results from the European project Fenix," IEEE Power Energy Soc. Gen. Meet., pp. 1-6, 2009 .

[4] P. Palensky and D. Dietrich, "Demand side management: Demand response, intelligent energy systems, and smart loads," IEEE Trans.
Ind. Informatics, vol. 7, no. 3, pp. 381-388, 2011.

[5] "No Title," http://www.smartenergydemand.eu. .

[6] A. Mnatsakanyan and S. W. Kennedy, "A Novel Demand Response Model with an Application for a Virtual Power Plant," IEEE Trans. Smart Grid, vol. PP, no. 99, pp. 1-1, 2014.

[7] A. Thavlov and H. W. Bindner, "Utilization of flexible demand in a virtual power plant set-up," IEEE Trans. Smart Grid, vol. 6, no. 2, pp. 640-647, 2015.

[8] "No Title," http://www.smarteremc2.eu/.

[9] CEN-CENELEC-ETSI Smart Grid Coordination Group, "Smart Grid Reference Architecture," no. November, p. 107, 2012.

[10] F. Andrén, T. Strasser, S. Rohjans, and M. Uslar, "Analyzing the need for a common modeling language for Smart Grid applications," IEEE Int. Conf. Ind. Informatics, pp. 440-446, 2013.

[11] N. Etherden, V. Vyatkin, and M. Bollen, "Virtual Power Plant for Grid Services using IEC 61850," IEEE Trans. Ind. Informatics, vol. 3203, no. c, pp. 1-1, 2015.

[12] R. Santodomingo, M. Uslar, A. Göring, M. Gottschalk, L. Nordström, A. Saleem, and M. Chenine, "SGAM-based methodology to analyze Smart Grid solutions," Energy Conf. (ENERGYCON), 2014 IEEE Int., pp. 751-758, 2014.

[13] J. Trefke, S. Rohjans, M. Uslar, S. Lehnhoff, L. Nordstrom, and a. Saleem, "Smart Grid Architecture Model use case management in a large European Smart Grid project," IEEE PES ISGT Eur. 2013, no. 978, pp. 1-5, 2013.

[14] H. S. Hippert, C. E. Pedreira, and R. C. Souza, "Neural networks for short-term load forecasting: a review and evaluation," IEEE Trans. Power Syst., vol. 16, no. 1, pp. 44-55, 2001.

[15] G. Sideratos and N. D. Hatziargyriou, "Probabilistic wind power forecasting using radial basis function neural networks," IEEE Trans. Power Syst., vol. 27, no. 4, pp. 1788-1796, 2012.

[16] J. Contreras, R. Espínola, F. J. Nogales, and A. J. Conejo, "ARIMA models to predict next-day electricity prices," IEEE Trans. Power Syst., vol. 18, no. 3, pp. 1014-1020, 2003.

[17] E. Mashhour and S. M. Moghaddas-Tafreshi, "Bidding strategy of virtual power plant for participating in energy and spinning reserve markets-Part I: Problem formulation," IEEE Trans. Power Syst., vol. 26, no. 2, pp. 949-956, 2011.

[18] M. Peik-Herfeh, H. Seifi, and M. K. Sheikh-El-Eslami, "Decision making of a virtual power plant under uncertainties for bidding in a day-ahead market using point estimate method," Int. J. Electr. Power Energy Syst., vol. 44, no. 1, pp. 88-98, 2013.

[19] IEADSM, "Market participant's views towards and experiences with Demand Side Bidding (Version 2)," vol. 44, no. January, p. Stage-1 Report (Version 2), 2002.

[20] S. Sučić, T. Dragičević, T. Capuder, and M. Delimar, "Economic dispatch of virtual power plants in an event-driven service-oriented framework using standards-based communications," Electr. Power Syst. Res., vol. 81, no. 12, pp. 2108-2119, 2011.

[21] A. Tascikaraoglu, O. Erdinc, M. Uzunoglu, and A. Karakas, "An adaptive load dispatching and forecasting strategy for a virtual power plant including renewable energy conversion units," Appl. Energy, vol. 119, pp. 445-453, 2014

[22] H. Yang, D. Yi, J. Zhao, and Z. Dong, "Distributed optimal dispatch of virtual power plant via limited communication," IEEE Trans. Power Syst., vol. 28, no. 3, pp. 3511-3512, 2013.

[23] J. Zapata, J. Vandewalle, and W. D'Haeseleer, "A comparative study of imbalance reduction strategies for virtual power plant operation," Appl. Therm. Eng., vol. 71, no. 2, pp. 847-857, 2014.

[24] A.L. Dimeas and N. D. Hatziargyriou, "Agent based control of virtual power plants." in Proc. 2007 IEEE Intelligent Systems Applications to Power Systems, pp. 1-6.

[25] R. Bienert, T. Avenue, and M. Hill, "OpenADR Profile Specification," pp. 1-106, 2013

[26] http://www.eurocontrol.int/services/weather-information-exchangemodel-wxxm.

[27] G. Messinis et al, "Multi-microgrid laboratory infrastructure for smart grid applications," 9th MedPower Coneference, Athens, Novemver 2014 